\title{
Treatment of the magnetic field for geodynamo simulations using the finite element method
}

\author{
Hiroaki Matsui ${ }^{1}$ and Hiroshi Okuda ${ }^{2}$ \\ ${ }^{1}$ Department of Geophysical Sciences, the University of Chicago, 5734 South Ellis Ave., Chicago, IL, 60637, USA \\ ${ }^{2}$ Department of Quantum Engineering and System Science, the University of Tokyo, \\ 7-3-1 Hongo, Bunkyo-ku, Tokyo 113-8656, Japan
}

(Received December 5, 2002; Revised August 10, 2004; Accepted August 30, 2004)

\begin{abstract}
We propose a scheme for calculating the magnetic field in a spherical shell, based on Earth's outer core, using the finite element method (FEM). The two most difficult problems for magnetohydrodynamics (MHD) simulations in a rotating spherical shell with FEM are solving the magnetic field outside the fluid shell, and connecting the magnetic field in the fluid shell to the exterior potential field at the boundary. To solve these problems, we extend the finite element mesh beyond the fluid shell and compute the vector potential of the magnetic field. To verify the present scheme, we consider three test case. First, we compare the FEM model with an analytical solution of Laplace's equation outside the fluid. Second, we evaluate free decay of a dipole field and compare the results with a spectral solution. Finally, compare the results of a simple kinematic dynamo problem with a spectral solution. The results suggest that the accuracy of the dipole field depends on the radius of the simulation domain, and that this error becomes sufficiently small if the radius of the outer region is approximately 6 times larger than the radius of the fluid shell.
\end{abstract}

Key words: Geodynamo, finite-element method, boundary conditions.

\section{Introduction}

It is widely accepted that the geomagnetic field is generated by the motion of an electrically conductive fluid in Earth's outer core, a phenomenon known as the geodynamo process. The fluid motion is strongly influenced by the Lorentz force and the Coriolis force, making the dynamo process a complicated nonlinear system that requires threedimensional, time-dependent numerical simulations. Investigations of the generation of magnetic fields for Earth and other planets entered a new phase in 1995 after several magnetohydrodynamic (MHD) simulations of a rotating spherical shell were used to represent some basic characteristics of the geomagnetic field (Glatzmaier and Roberts, 1995a, b; Kageyama et al., 1995). Following this work, many simulation studies of the geodynamo represented the strong and dipole-like magnetic fields by which the geomagnetic field is characterized (Kuang and Bloxham, 1997; Christensen et al., 1999; Sakuraba and Kono, 1999). However, most of these simulations utilize spherical harmonic expansions in the azimuthal and elevation directions. This choice was made because of the high spectral accuracy of this method and because the magnetic fields in the spherical shell are easily connected to the potential field outside the shell at the boundary. Kageyama et al. (1995) used the finite difference method (FDM), but incorporated a magnetic boundary condition at the shell boundaries that is different to that assumed to exist at Earth's Core-Mantle boundary (CMB).

Copy right (c) The Society of Geomagnetism and Earth, Planetary and Space Sciences (SGEPSS); The Seismological Society of Japan; The Volcanological Society of Japan; The Geodetic Society of Japan; The Japanese Society for Planetary Sciences; TERRAPUB.
As noted, the use of spherical harmonic expansions has appealing advantages for geodynamo simulations, but it nevertheless has some disadvantages. In particular, the spherical harmonics expansion is not suitable for massive parallel computations because a significant number of global operations are required for the computation of nonlinear terms. Kuang and Bloxham (1997) sub-divided the spherical shell radially. However, the number of the processors used for the simulation limits the model's radial resolution. Clune et al. (1999) introduced a method of parallel computation for fluid motion in a rotating spherical shell using a spherical harmonics expansion. Their scheme requires three decompositions of the model domain for time integration. Several other schemes for simulating the fluid motion exist, namely the finite-volume method (FVM), and the finite-element method (FEM). These schemes are more suitable for parallel computation because they consist of local operations. For the present study, we choose the finite element method. Under the FEM, any unstructured mesh can be used-that is, one that is suitable for describing some complex geometry at the CMB. Furthermore, local diffusivities are easily introduced under the FEM platform because the operations are local. However, the following problems remain to be solved:

- Solving for the magnetic field using the induction equation and the magnetic field conservation law. There are four equations for the three components of the field.

- Connecting the magnetic field in the fluid shell to the magnetic field outside the shell. 
- Determining the magnetic diffusion term that satisfies the magnetic boundary condition on the CMB.

Chan et al. (2001a, b) solved a nonlinear kinematic dynamo problem using the FEM platform. They used a finite-element mesh not only for the fluid shell, but also for the exterior of the shell, and they introduced an auxiliary pressure to the magnetic induction equation, to solve for both the magnetic field and the auxiliary pressure. The approach we adopt to determine the magnetic field is different from that of Chan et al. The principal differences between the two codes are as follows:

- In the present model, the simulation domain is divided into hexahedral elements, and all physical values including the potential $\varphi$ are interpolated by tri-linear functions. On the other hand, Chan et al. used quadratic shape functions to interpolate the magnetic field, and a linear function to interpolate the auxiliary pressure in tetrahedral elements.

- In the present model, the vector potential is solved for, whereas the magnetic field is calculated in Chan et al.'s model.

- In our model, the exterior of the fluid shell is assumed to be an insulator and is represented by the Laplace equation, whereas Chan et al. assume that a region of large magnetic diffusivity extends beyond the fluid shell.

As with the approach of Chan et al., a finite-element mesh is required for both the fluid shell and the shell's exterior, but we can treat electrical insulators in the present model. Here we propose a method based on the vector potential of the magnetic field to perform geodynamo simulations in a rotating spherical shell. We verify the present scheme with three simple tests. The first test is, given a vector potential in the fluid shell, to determine the potential magnetic field outside the shell in order to investigate the required size of the simulation domain. In the second test, the magnetic diffusion in the fluid shell is computed and compared the results with a spectral solution. Thirdly, a simple kinematic dynamo problem is solved and the results are compared with those obtained using a spherical harmonics expansion.

\section{Simulation Model and Methods}

\subsection{Basic equations for the magnetic field}

Consider a spherical shell modeled on Earth's outer core. The ratio of the inner boundary to the outer boundary of the spherical shell is set to be 0.4. The shell is filled with an electrically conducting fluid of constant conductivity $\sigma$. In the case of the kinematic dynamo problem, the fluid has a given velocity field $\boldsymbol{u}$. The basic equations for the magnetic field, under the MHD approximation, are given by Maxwell's equations and Ohm's law-that is:

$$
\begin{aligned}
\nabla \times \boldsymbol{E} & =-\frac{\partial \boldsymbol{B}}{\partial t}, \\
\nabla \times \boldsymbol{B} & =\mu_{0} \boldsymbol{J}, \\
\nabla \cdot \boldsymbol{E} & =\frac{\rho_{e}}{\epsilon_{0}}, \\
\nabla \cdot \boldsymbol{B} & =0,
\end{aligned}
$$

and

$$
\boldsymbol{J}=\sigma(\boldsymbol{E}+\boldsymbol{u} \times \boldsymbol{B}),
$$

where $\boldsymbol{B}, \boldsymbol{E}, \boldsymbol{J}, \mu_{0}$, and $\rho_{e}$ are the magnetic field, electric field, current density, magnetic permeability, and charge density, respectively. The vector potential of the magnetic field $\boldsymbol{A}$ with the Coulomb gauge is considered in the present formulation. The vector potential satisfies the following equations;

$$
\nabla \times \boldsymbol{A}=\boldsymbol{B}
$$

and

$$
\nabla \cdot \boldsymbol{A}=0 .
$$

Using Eqs. (1)-(7), the basic equations for the vector potential are found to be;

$$
\begin{aligned}
\frac{\partial \boldsymbol{A}}{\partial t} & =-\nabla \varphi+\eta \nabla^{2} \boldsymbol{A}+\boldsymbol{u} \times \boldsymbol{B}, \\
\nabla \cdot \boldsymbol{A} & =0,
\end{aligned}
$$

and

$$
\nabla \times \boldsymbol{A}=\boldsymbol{B}
$$

where $\eta=1 /\left(\mu_{0} \sigma\right)$ is the magnetic diffusivity, and $\varphi$ is an scalar potential.

These basic equations for $\boldsymbol{A}$ and $\varphi$ (Eqs. (8) and (9)) are similar to the basic equations for the velocity and pressure of a Boussinesq fluid. Consequently, the same time integration scheme can be applied for the vector potential as that for the fluid motion.

A characteristic of the present simulation is that the vector potential has to be determined outside the radius of the fluid shell, which we denote by $r_{o}$. The solution for the magnetic field in the insulating region $\left(r>r_{o}\right)$ can be described by a potential field

$$
\boldsymbol{B}=-\nabla W,
$$

where the potential $W$ is expanded in spherical harmonics $Y_{l}^{m}$ as

$$
W=\sum_{l=1}^{\inf } \sum_{m=-l}^{l}\left(\frac{r_{o}}{r}\right)^{l+1} C_{l}^{m} Y_{l}^{m},
$$

and $C_{l}^{m}$ are the scalar coefficients of the expansion. In a spectral method, the magnetic boundary condition can be given as Neumann boundary conditions for each scalar coefficient in the expansion. However, we cannot apply such boundary conditions here because the FEM is based on local operations. Following Chan et al. (2001b), the finite element mesh is extended outside the fluid shell. The basic equations for the vector potential in the insulator are

$$
J_{e}=-\nabla^{2} \boldsymbol{A}_{e}=0,
$$

and

$$
\nabla \cdot \boldsymbol{A}_{e}=0,
$$

where $\boldsymbol{A}_{e}$ and $\boldsymbol{J}_{e}$ are the vector potential and current density in the insulator, respectively. In this formulation, Eqs. (8) and (13) can be solved simultaneously because physical properties can be defined for each element under the FEM platform. 


\subsection{Boundary conditions}

The boundary conditions for the vector potential at the fluid-insulator interface are specified by continuity of the magnetic field and tangential components of the electric field. The boundary conditions for the magnetic field are

$$
\left(\boldsymbol{B}-\boldsymbol{B}_{e}\right) \cdot \hat{n}=0,
$$

and

$$
\left(\boldsymbol{B}-\boldsymbol{B}_{e}\right) \times \hat{n}=0,
$$

where $\boldsymbol{B}, \boldsymbol{B}_{e}$, and $\hat{n}$ are the magnetic field in the fluid shell and the insulator, and the normal vector of the boundary, respectively. To satisfy these boundary conditions, we require the following conditions on the vector potential,

$$
\left(\frac{\partial \boldsymbol{A}}{\partial n}-\frac{\partial \boldsymbol{A}_{e}}{\partial n}\right)=0
$$

and

$$
\left(\boldsymbol{A}-\boldsymbol{A}_{e}\right)=0
$$

The boundary condition on the electric field requires

$$
\begin{aligned}
\left(\boldsymbol{E}-\boldsymbol{E}_{e}\right) \times \hat{n}= & -\left(\frac{\partial \boldsymbol{A}}{\partial t}-\frac{\partial \boldsymbol{A}_{e}}{\partial t}\right) \times \hat{n} \\
& -\left(\nabla \varphi-\nabla \varphi_{e}\right) \times \hat{n}=0,
\end{aligned}
$$

where, $\boldsymbol{E}_{e}$ is the electric field in the insulator. In order to ensure that $\nabla \varphi$ is define on the boundary, we require $\varphi$ to be continuous across the boundary. This requires

$$
\varphi-\varphi_{e}=0
$$

and

$$
\left(\nabla \varphi-\nabla \varphi_{e}\right) \times \hat{n}=0 .
$$

These boundary conditions (Eqs. (17)-(18), (20), and (21)) are easily implemented under the FEM platform. There are no conditions on the normal component of $\boldsymbol{E}$. Therefore,

$$
\begin{aligned}
\left(\boldsymbol{E}-\boldsymbol{E}_{e}\right) \cdot \hat{n}= & -\left(\frac{\partial \boldsymbol{A}}{\partial t}-\frac{\partial \boldsymbol{A}_{e}}{\partial t}\right) \cdot \hat{n} \\
& -\left(\frac{\partial \varphi}{\partial n}-\frac{\partial \varphi_{e}}{\partial n}\right) \cdot \hat{n} \neq 0
\end{aligned}
$$

Since $\left(\partial \boldsymbol{A} / \partial t-\partial \boldsymbol{A}_{e} / \partial t\right)=0$ by virtue of Eq. (18), the discontinuity of $\boldsymbol{E} \cdot \hat{n}$ is due to a discontinuity in the normal gradient of the scalar potential $\partial \varphi / \partial n$. As a result $\partial \varphi / \partial n$ is discontinuous at the boundary when the conductivity changes across the boundary.

We should impose boundary conditions at the infinite radius. In the present study, the following boundary condition should be considered at infinity;

$$
\boldsymbol{A}_{e}=O\left(r^{-2}\right)
$$

However, because we are dealing with a finite area divided into a finite number of elements, we apply a modified boundary condition,

$$
\boldsymbol{A}_{e}=0,
$$

at the limit of the finite-element mesh $r=r_{m}$ in place of Eq. (23). To approach the real solution of the vector potential near the outer boundary of the fluid shell, $r_{m}$ should be as large as possible. Furthermore, a boundary condition for the scalar potential $\varphi_{e}$ is also required at $r=r_{m} . \varphi_{e}$ also approaches zero at the infinite radius. However, the following condition is used as the boundary condition to satisfy the Coulomb gauge, at $r=r_{m}$ :

$$
\frac{\partial \varphi_{e}}{\partial n}=0
$$

\subsection{Time integration scheme}

The present code is based on the thermal-hydraulic subsystem of GeoFEM, which serves a parallel FEM platform (Matsui and Okuda, 2002). We use the fractional step scheme to solve for the vector potential, by solving Eqs. (8) and (13) simultaneously. The Crank-Nicolson scheme is used to determine the diffusion term, and the 2nd-order Adams-Bashforth Scheme is used to find the induction term in the time integration process. The potential $\varphi$ and diffusion term are solved using the Conjugate Gradient solver by GeoFEM (Nakajima and Okuda, 1999).

The processes of time integration is described as follows:

$$
\begin{aligned}
\frac{1}{\Delta t}\left(\boldsymbol{A}^{n+\frac{1}{3}}-\boldsymbol{A}^{n}\right) & =\frac{3}{2}\left(\boldsymbol{u}^{n} \times \boldsymbol{B}^{n}\right) \\
& -\frac{1}{2}\left(\boldsymbol{u}^{n-1} \times \boldsymbol{B}^{n-1}\right), \\
\frac{1}{\eta \Delta t} \boldsymbol{A}^{n+\frac{2}{3}}-\frac{1}{2} \nabla^{2} \boldsymbol{A}^{n+\frac{2}{3}} & =\frac{1}{\eta \Delta t} \boldsymbol{A}^{n+\frac{1}{3}}+\frac{1}{2} \nabla^{2} \boldsymbol{A}^{n}, \\
-\Delta t \nabla^{2} \varphi^{n+1} & =-\nabla \cdot \boldsymbol{A}^{n+\frac{2}{3}},
\end{aligned}
$$

and

$$
\boldsymbol{A}^{n+1}=\boldsymbol{A}^{n+\frac{2}{3}}-\Delta t \nabla \varphi^{n+1}
$$

Within the insulator, Eqs. (13) and (14) should be solved. To solve these equations, we use the same treatment as that for Eqs. (27)-(29). The time integration is given by

$$
\begin{aligned}
\frac{1}{2} \nabla^{2} \boldsymbol{A}_{e}^{n+\frac{2}{3}} & =\frac{1}{2} \nabla^{2} \boldsymbol{A}_{e}^{n}(=0), \\
-\Delta t \nabla^{2} \varphi_{e}^{n+1} & =-\nabla \cdot \boldsymbol{A}_{e}^{n+\frac{2}{3}},
\end{aligned}
$$

and

$$
\boldsymbol{A}_{e}^{n+1}=\boldsymbol{A}_{e}^{n+\frac{2}{3}}-\Delta t \nabla \varphi_{e}^{n+1}
$$

By this procedures, the current density at the next step satisfies $\boldsymbol{J}_{e}^{n+1}=\nabla \times \nabla \times \boldsymbol{A}_{e}^{n+2 / 3}=\nabla\left(\nabla \cdot \boldsymbol{A}_{e}^{n+2 / 3}\right)$. The current density $\boldsymbol{J}_{e}^{n+1}$ is not zero because $\nabla \cdot \boldsymbol{A}_{e}^{n+2 / 3} \neq 0$. However, the magnetic field is not affected by the error of $\boldsymbol{A}_{e}^{n+2 / 3}$ because this error consists of a potential field (see Eq. (31)). The current density $\boldsymbol{J}_{e}^{n+1}$ is due to a change of the charge density on the boundary between the insulator and conductive fluid, and is neglected under the MHD approximation.

The simulation domain is divided into tri-linear hexahedral elements. The vector potential, magnetic field, scalar potential, velocity, and temperature are defined at each node and interpolated by a tri-linear function $N_{\beta}$ in each element. 


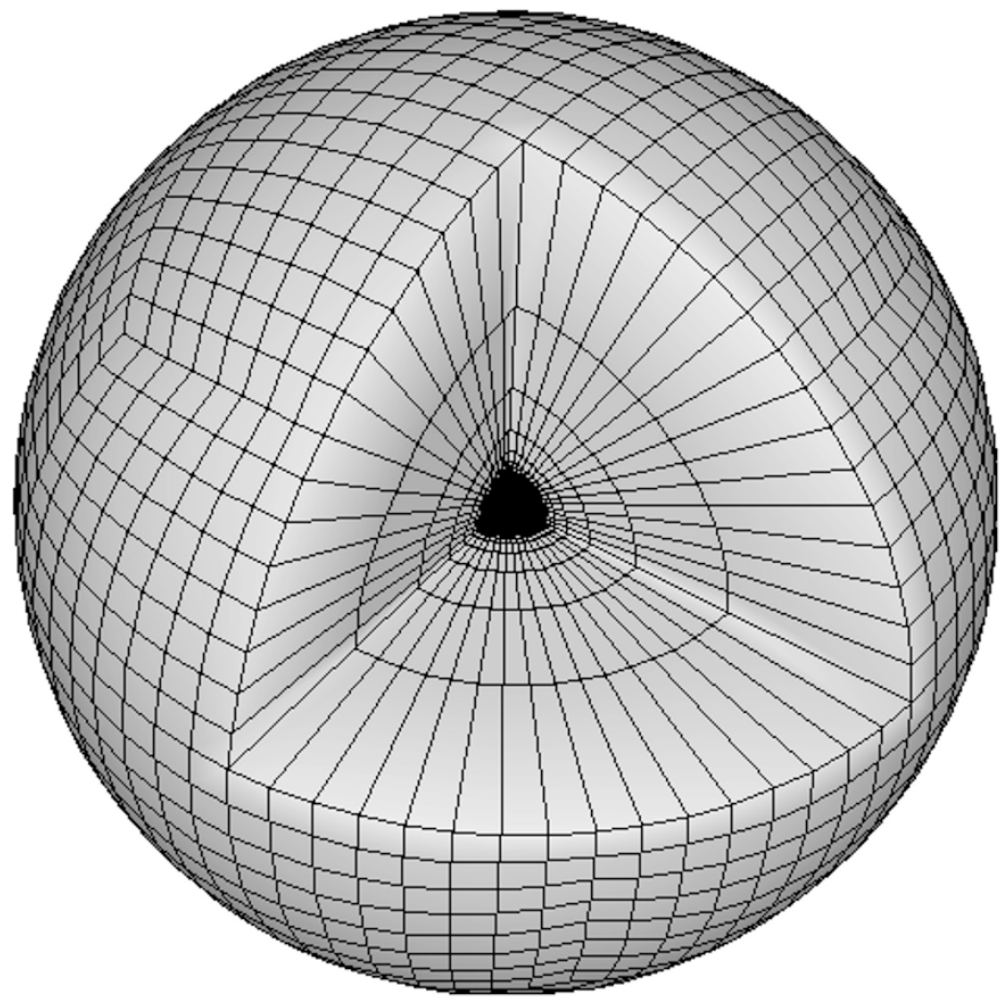

Fig. 1. Finite element mesh for the present model. This finite element mesh consists of $1.48 \times 10^{5}$ nodes, and the size of the simulation domain in the present figure is $r_{m}=17.6$.

The suffix for insulator $e$ is omitted in the following equations. To describe the time evolution scheme in the weak form, Eqs. (26) and (27) are multiplied by eight weighting functions $N_{\alpha}$. By connecting these equations in the conductive fluid and the insulator, the following matrix equations are obtained:

$$
\begin{aligned}
& \sum_{\text {Fluid }} \sum_{\alpha} \sum_{\beta} \bar{M}_{\alpha \beta}\left(\boldsymbol{A}_{\beta}^{n+\frac{1}{3}}-\boldsymbol{A}_{\beta}^{n}\right) \\
= & \Delta t \sum_{\text {Fluid }} \sum_{\alpha} \sum_{\beta}\left(\frac{3}{2} \boldsymbol{f}_{A}^{n}-\frac{1}{2} \boldsymbol{f}_{A}^{n-1}\right) \\
& \sum_{\text {Fluid }} \sum_{\alpha} \sum_{\beta} \frac{1}{\eta \Delta t} \bar{M}_{\alpha \beta}\left(\boldsymbol{A}_{\beta}^{n+\frac{2}{3}}-\boldsymbol{A}_{\beta}^{n+\frac{1}{3}}\right) \\
= & \frac{1}{2}\left[\sum_{\text {Fluid }} \sum_{\alpha} \sum_{\beta}\left(S_{\alpha \beta}-L_{\alpha \beta}\right)\left(\boldsymbol{A}_{\beta}^{n+\frac{2}{3}}+\boldsymbol{A}_{\beta}^{n}\right)\right. \\
& \left.+\sum_{\text {Ins }} \sum_{\alpha} \sum_{\beta}\left(S_{\alpha \beta}-L_{\alpha \beta}\right)\left(\boldsymbol{A}_{\beta}^{n+\frac{2}{3}}+\boldsymbol{A}_{\beta}^{n}\right)\right], \\
& -\Delta t \sum_{\text {Entire }} \sum_{\alpha} \sum_{\beta}\left(S_{\alpha \beta}-L_{\alpha \beta}\right) \varphi_{\beta}^{n+1} \\
= & -\sum_{\text {Entire }} \sum_{\alpha} \sum_{\beta} \boldsymbol{H}_{\alpha \beta} \cdot \boldsymbol{A}_{\beta}^{n+\frac{2}{3}},
\end{aligned}
$$

and

$$
\sum_{\text {Entire }} \sum_{\alpha} \sum_{\beta} \bar{M}_{\alpha \beta}\left(\boldsymbol{A}_{\beta}^{n+1}-\boldsymbol{A}_{\beta}^{n+\frac{2}{3}}\right)
$$

$$
=-\sum_{\text {Entire }} \sum_{\alpha} \sum_{\beta} \boldsymbol{H}_{\alpha \beta} \varphi^{n+1},
$$

where

$$
\boldsymbol{f}_{A}^{n}=M_{\alpha \beta} \boldsymbol{u}_{\beta}^{n} \times \boldsymbol{B}_{e}^{n} .
$$

In the above equations, $\boldsymbol{B}_{e}=V_{e}^{-1} \sum_{\alpha} \int_{e} B_{\alpha} N_{\alpha} d V$ is the magnetic field averaged over each element, and $\sum_{\text {Fluid }}$, $\sum_{\text {Ins }}$, and $\sum_{\text {Entire }}$ indicate integration in the fluid core, the insulator, and the entire domain, respectively. The integrations $M_{\alpha, \beta}, L_{\alpha \beta}, S_{\alpha \beta}, H_{\alpha \beta}$, and $\bar{M}_{\alpha \beta}$ are given by;

$$
\begin{aligned}
M_{\alpha \beta} & =\int_{e} N_{\alpha} N_{\beta} d V, \\
L_{\alpha \beta} & =\int_{e}\left\{\left(\nabla N_{\alpha}\right) \cdot\left(\nabla N_{\beta}\right)\right\} d V, \\
S_{\alpha \beta} & =\oint_{e} N_{\alpha}\left(\nabla N_{\beta}\right) \cdot \hat{n} d S, \\
\boldsymbol{H}_{\alpha \beta} & =\int_{e} N_{\alpha}\left(\nabla N_{\beta}\right) d V,
\end{aligned}
$$

and

$$
\bar{M}_{\alpha \beta}=\left\{\begin{array}{c}
\sum_{\beta} M_{\alpha \beta}, \alpha=\beta \\
0, \quad \alpha \neq \beta
\end{array}\right.
$$

It is noted that the surface integration term at the CMB in Eq. (34) can be omitted because of the boundary condition for the vector potentialin Eq. (17). 


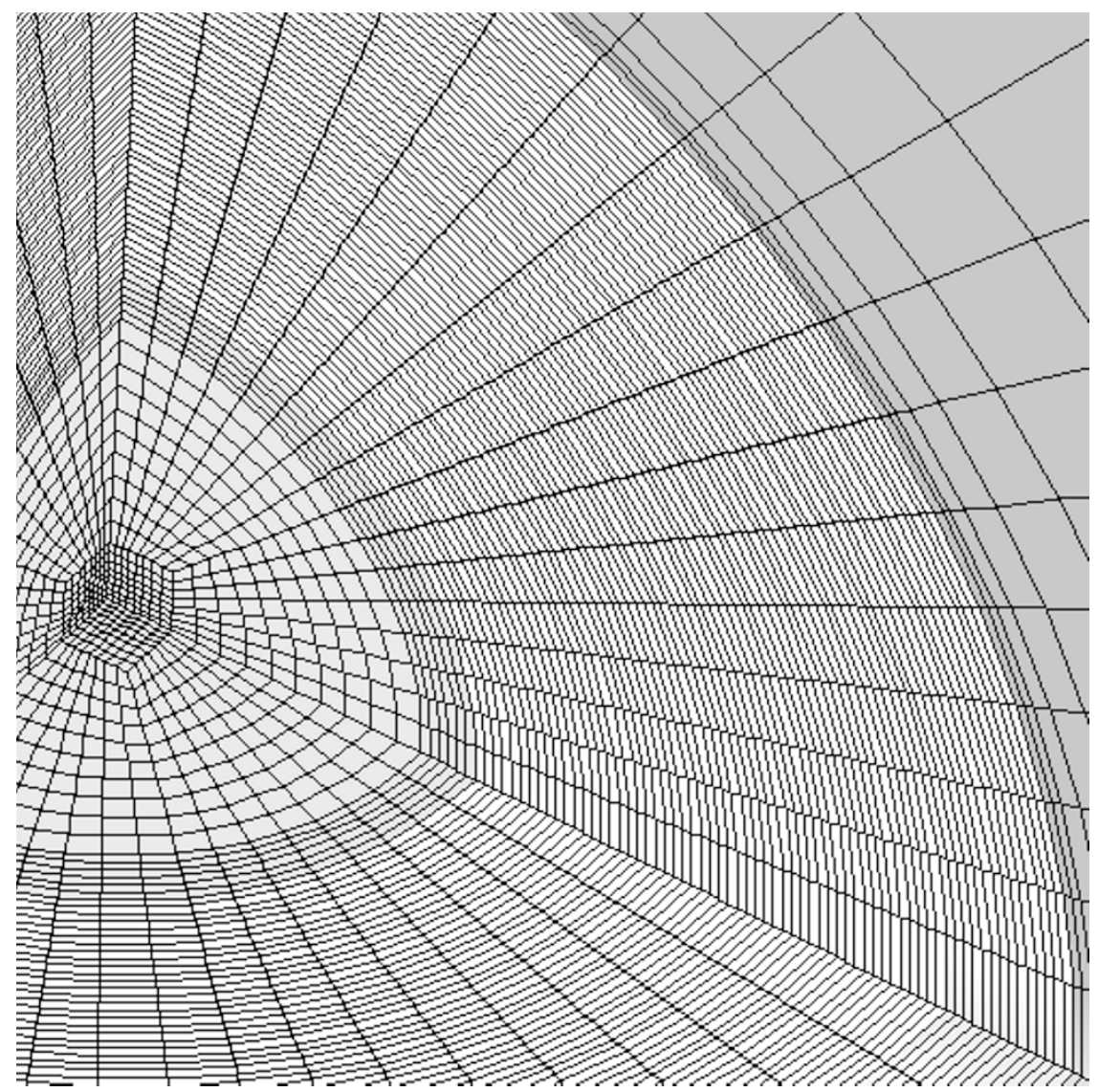

Fig. 2. Mesh pattern for the fluid shell given in Fig. 1. The finite elements for the outer core are white: the elements for the inner core and outside the shell are gray.

\subsection{Finite element mesh}

The simulation domain is divided into tri-linear hexahedral elements, as illustrated in Fig. 1. The radius of the simulation domain $r_{m}$ is varied to investigate the effects of size on results. The mesh is constructed as follows.

- The sphere's surface is divided into quadrilateral elements, which are stacked radially throughout most of the problem domain.

- A divided cube is used at the model's center.

- Connecting elements link the outer spherical shell and the inner cubic mesh.

In the present study, the outer core is equally divided in the radial direction, while the element sizes increase with radial distance outside the core (see Fig. 1). As seen in Fig. 2, the mesh becomes somewhat irregular around the cubic mesh. These grid patterns may affect the simulation results, but any such effects will be small because the magnetic field patterns are simple near the center.

\subsection{Parallelization}

We performed the present simulations on a Hitachi SR8000, which has eight computational nodes, where each SMP node consists of eight processors. We choose OpenMP for parallelization in each SMP node, and a domain decomposition method for parallelization among SMP nodes. There is a problem associated with the domain decomposition in the present study, because the number of computa-

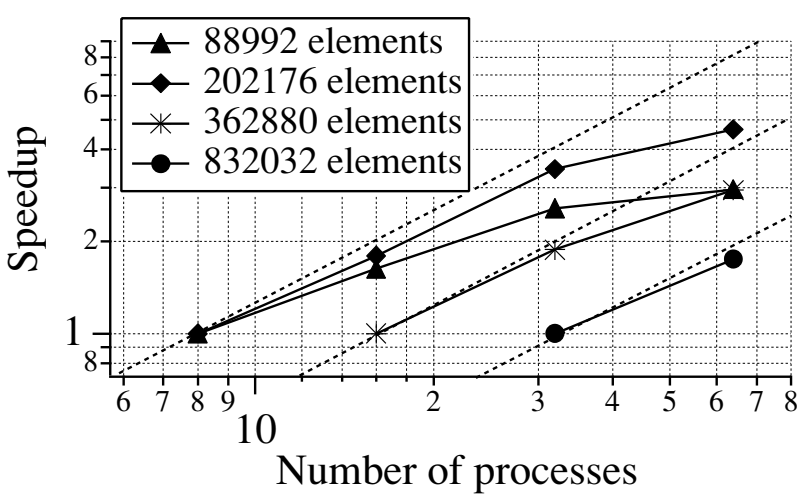

Fig. 3. Parallel performance on a SR8000 system. The speedup is calculated from the elapsed time for 10 time steps.

tions is different for the conductive fluid and for the insulator. To balance the computation for each domain, the simulation domain is divided along azimuthal and elevation directions.

Figure 3 shows the parallel efficiency for 10 time steps on the SR8000 using four different spatial resolutions. We find that the parallel efficiency decreases when the number of elements for each processor is small, because the ratio of the communication time to computation time becomes large in this case. This suggests that approximately $10^{5}$ elements/(SMP node) are required to obtain an adequate parallel performance on the SR8000. 
Table 1. Spatial resolution of the finite element mesh.

\begin{tabular}{c|ccc}
\hline Name & $\Delta r$ & Num. of layer & $r_{m}$ \\
& for fluid & in $r_{o}<r<r_{m}$ & \\
\hline Mesh (a) & $1 / 32$ & $7 \sim 9$ & $5.635 \sim 16.635$ \\
Mesh (b) & $1 / 64$ & $9 \sim 11$ & $5.667 \sim 17.667$ \\
Mesh (c) & $1 / 128$ & $8 \sim 12$ & $2.667 \sim 17.667$ \\
\hline
\end{tabular}

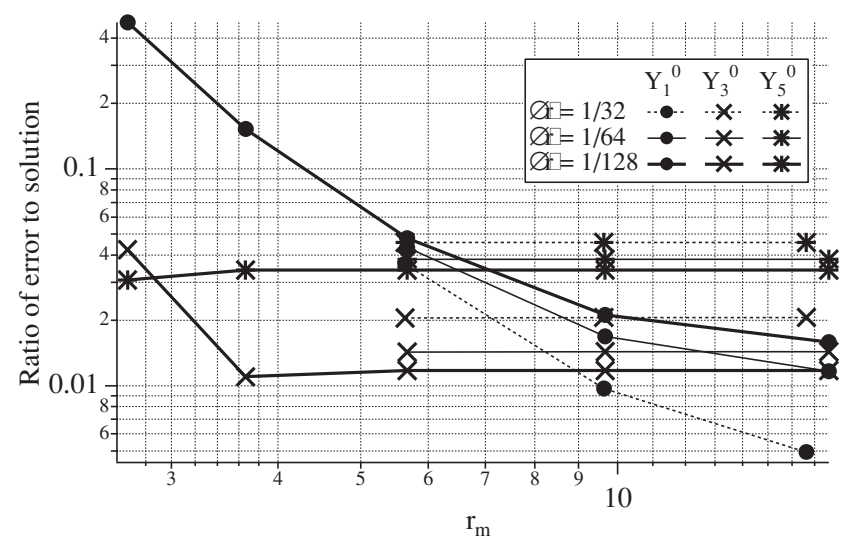

Fig. 4. Ratio of the R.M.S. error in the magnetic field to the R.M.S. of the magnetic field at $r=r_{o}$ as a function of the radius of the simulation domain $r_{m}$.

\section{Results of Test Simulations}

Three tests are performed to evaluate the present simulation model:

- Analyze the vector potential outside the fluid shell to examine the effects of different maximum simulation radii.

- Solve the magnetic diffusion equation for an initial dipole field.

- Solve a simple kinematic dynamo problem and comparing the solution with the results of a spherical harmonics expansion.

\subsection{Accuracy tests for a magnetic field in the insulating mantle}

3.1.1 Effect of the radial resolution and size of simulation domain Here we focus on the calculation of a magnetic field outside the fluid shell and investigate its accuracy. The magnetic vector potential is obtained by solving a boundary problem (Eqs. (13) and (14) in the insulating domain. In this test we used the following magnetic field for the fluid shell.)

$$
\begin{aligned}
\boldsymbol{A} & =\nabla \times\left(B_{S l}^{m} Y_{l}^{m} \hat{r}\right), \\
\boldsymbol{B} & =\nabla \times \nabla \times\left(B_{S l}^{m} Y_{l}^{m} \hat{r}\right), \\
B_{S l}^{0} & =\left\{\begin{array}{clc}
B_{S l}^{0}\left(r=r_{i}\right)\left(\frac{r}{r_{i}}\right)^{l+1} & \text { for } \quad r<r_{i} \\
\cos \left(\alpha_{l} r-\beta_{l}\right) & \text { for } r_{i} \leq r \leq r_{o} \\
B_{S l}^{0}\left(r=r_{o}\right)\left(\frac{r_{o}}{r}\right)^{l} & \text { for } \quad r>r_{o}
\end{array}\right.
\end{aligned}
$$

Table 2. Number of node and element of the finite-element mesh.

\begin{tabular}{c|ccc}
\hline Name & $k_{\text {grid }}$ & $\begin{array}{c}\text { Num. of layer } \\
\text { in } r_{o}<r<r_{m}\end{array}$ & Range of $r_{m}$ \\
\hline Mesh (d) & 1.2 & $21 \sim 30$ & $4.599 \sim 17.058$ \\
Mesh (e) & 1.5 & $13 \sim 17$ & $5.706 \sim 22.177$ \\
Mesh (f) & 2.0 & $9 \sim 11$ & $5.667 \sim 17.667$ \\
Mesh (g) & 2.5 & $7 \sim 9$ & $4.215 \sim 17.57$ \\
\hline
\end{tabular}

where, $Y_{l}^{m}$ is the spherical harmonic, and $\alpha_{l}$ and $\beta_{l}$ are chosen to satisfy the following conditions;

$$
\begin{gathered}
\alpha_{l} \tan \left(\alpha_{l} r_{i}-\beta_{l}\right)=-\frac{l+1}{r_{i}}, \\
\alpha_{l} \tan \left(\alpha_{l} r_{o}-\beta_{l}\right)=\frac{l}{r_{o}} .
\end{gathered}
$$

This magnetic field connects to the field in the insulated domain. The vector potential is given in the fluid shell, and the vector potential outside the shell is solved by matching the field on the boundaries of the shell. In the present test, $(l, m)=(1,0),(3,0)$, and $(5,0)$ is used, and the numerical solution for the magnetic field intensity is compared with the analytical solution.

As summarized in Table 1, three different radial resolutions were considered, and a different size of simulation domain was used for each mesh. We fixed the horizontal resolution in this test by setting the number of elements in each sphere to 1944 . The angular distance between each node at the equatorial plane was $5^{\circ}$. The thickness of the elements $\Delta r$ outside the fluid shell was set as follows:

- The same $\Delta r$ as that in the fluid shell is used for the next two layers to the outer boundary of the shell.

- For the other layers, $\Delta r$ at $n$-th layer $\Delta r_{n}$ is defined by the ratio $k_{\text {grid }}=\Delta r_{n} / \Delta r_{n-1}=2$.

The modeling error is estimated in terms of the intensity of the magnetic field at the outer boundary of the fluid shell. The ratio of the root mean square (R.M.S.) of the error in the magnetic field to the R.M.S. of the magnetic field intensity is plotted in Fig. 4. Only the dipole component $\left(Y_{1}^{0}\right)$ has a dependence on $r_{m}$ when $r_{m}>5.6$. The accuracy of the higher components depends on the radial resolution of the finite element mesh. As seen from Eq. (45), the $Y_{3}^{0}$ and $Y_{5}^{0}$ components approach zero rapidly. To obtain accurate solutions for these higher degrees of the magnetic field, the spatial resolution near the outer boundary of the fluid shell is more important than the total size of the simulation domain. In contrast, the accuracy of the dipole magnetic field has a clear dependence on $r_{m}$. It is noted that only the dipole component tends to have large errors when the radial resolution is good, suggesting that the accuracy of component is limited by the size of the simulation domain. In the present case, the error for the dipole component is $2.1 \%$ when $r_{m}=9.667$ in Mesh (c). We consider this level of error to be acceptable given the spatial resolution. 


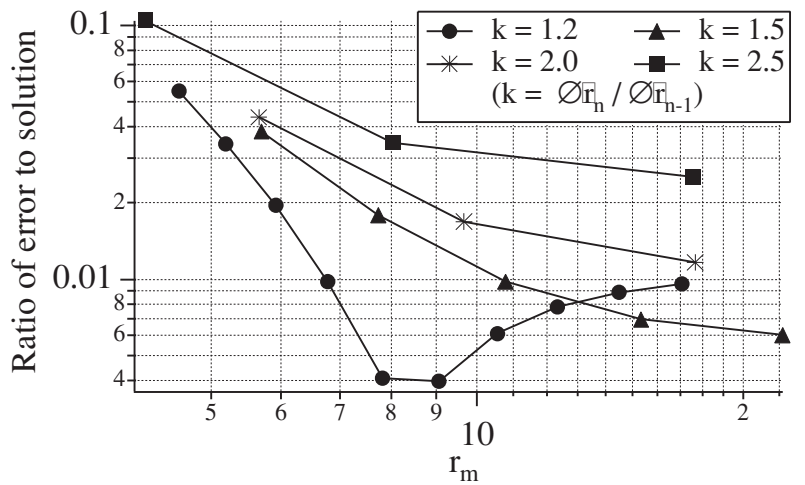

Fig. 5. The same plot as Fig. 4 for investigation of dependence on $k_{\text {grid }}$.

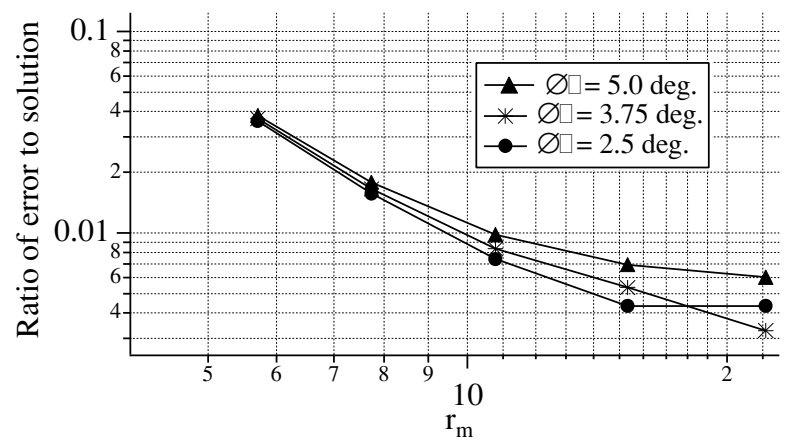

Fig. 6. The same plot as Fig. 4 for investigation of dependence on horizontal resolution.

3.1.2 Effect of the radial mesh pattern in the insulator Next we investigate the effect of the mesh pattern outside the shell by changing the ratio of the width of the adjacent elements $k_{\text {grid }}=\Delta r_{n} / \Delta r_{n-1}$. We changed the ratio $k_{\text {grid }}$ outside the fluid shell in Mesh (b), as described in the previous section (see Table 2). The ratio of the R.M.S. error in the analytical solution to the R.M.S. of the analytical solution for the axial dipole component was estimated in the present test. The results are plotted in Fig. 5. The errors are reduced by using small $k_{\text {grid }}$ except for $k_{\text {grid }}=1.2$ cases. The error converges to $1 \%$ for the analytical solution with large $r_{m}$. We attribute this error to the horizontal discretization. The magnitude of this error in this case is similar to that in the case of $r_{m}>10$ with $k_{\text {grid }}=2$.

3.1.3 Effect of horizontal resolution Finally, we estimated the error due to horizontal resolution. We chose Mesh (e) from the previous test, and changed the horizontal resolution at the equatorial plane from $5.0^{\circ}$ to $3.75^{\circ}$ and $2.5^{\circ}$. The results are plotted in Fig. 6. As seen in Fig. 6, the obtained accuracy decreases with the horizontal resolution. The results suggest that the horizontal resolution is less important for the dipole field than both the size of the simulation domain and the radial resolution outside the fluid shell.

\subsection{Accuracy test of magnetic diffusion}

In this test, we set $\eta=1.0$ and $\boldsymbol{u}=0$ in Eqs. (8)-(10). In the present simulation, we can maintain numerical stability for the diffusion term because the Crank-Nicolson scheme is used for the diffusion term. However, we set the length of each time step to be less than $1 /(\Delta r)^{2}$, where $\Delta r$ is the
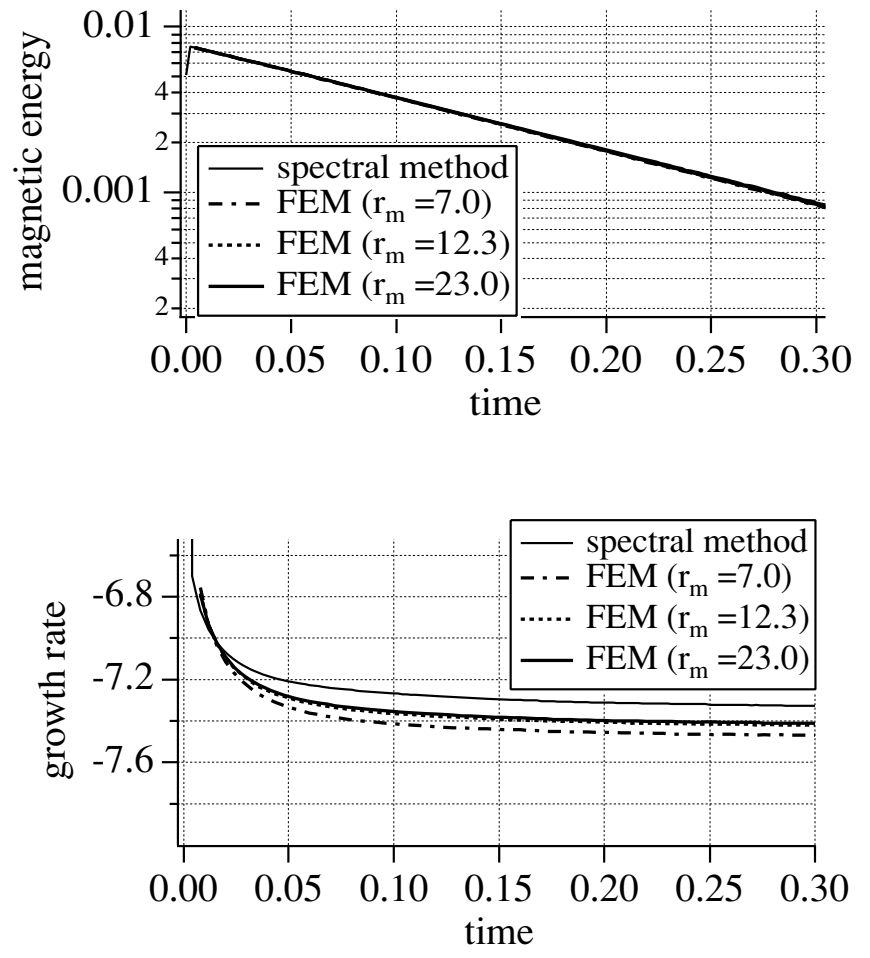

Fig. 7. Time evolutions of the averaged magnetic energy (upper panel) and growth rate of the magnetic energy (lower panel).

width of elements in the fluid shell, as follows:

$$
\Delta t=2.5 \times 10^{-3} \text {. }
$$

As an initial value, we use an axial dipole field given by Eqs. (43)-(45) with $l=1$. The finite element mesh configuration for the present test is given in Table 3; we performed the test with three simulation domain sizes $r_{m}$. In order to verify the results, the same computation was performed using a spherical harmonics expansion in the azimuthal and elevation directions, and a finite-difference method in the radial direction. In this scheme, 49 equally spaced grid points were used in the radial direction. As seen in the upper panel of Fig. 7, the magnetic energy averaged over the fluid shell $E_{\text {mag }}$ decays exponentially in all cases. The growth rate of the magnetic energy $k$ given by $E_{\text {mag }}(t) / E_{\text {mag }}(t-\Delta t)=$ $\exp (k \Delta t)$ is plotted in the lower panel of Fig. 7. The growth rate of the magnetic energy approaches a constant value in each case, and this value is almost the same in the cases with $r_{m}=12.3$ and $r_{m}=23.4$. The averaged magnetic energy and the growth rate for $t=0.3$ are given in Table 4. The growth rate for the case of $r_{m}=12.3$ differs by only $0.13 \%$ from that for $r_{m}=23.0$, while the error between these cases and the spectral method is $6.4 \%$. The discrepancy between the FEM results and the spectral method result is presumably related to spatial resolution in the azimuthal and elevation directions for the FEM.

\subsection{Accuracy test of kinematic dynamo problem}

The velocity field of the conductive fluid is prescribed in this problem. Because the problem is linear, a significant number of studies of the kinematic dynamo problem have been reported since the work of Bullard and Gillman (1954). We consider a modified model of Lilley's dynamo (Lilley, 
Table 3. Resolution of the finite element mesh.

\begin{tabular}{|c|c|c|c|c|c|}
\hline \multirow[t]{2}{*}{ Name } & \multicolumn{3}{|c|}{$N_{\text {element }}$} & \multirow{2}{*}{$\begin{array}{c}\Delta r \\
\text { for fluid }\end{array}$} & \multirow[t]{2}{*}{$r_{m}$} \\
\hline & For inner core & For fluid & For external & & \\
\hline Mesh (h) & 42768 & 93312 & $15552 \sim 19440$ & $1 / 48$ & $5.00 \sim 23.0$ \\
\hline
\end{tabular}

Table 4. Magnetic energy $E_{\text {mag }}$, its growth rate $k$, local magnetic field, and maximum zonal current density for $t=0.3$.

\begin{tabular}{c|ccccc}
\hline$r_{m}$ & $E_{\text {mag }}$ & $k$ & $B_{z}$ at $\boldsymbol{r}=\left(0,0, r_{o}\right)$ & $B_{z}$ at $\boldsymbol{r}=\left(r_{o}, 0,0\right)$ & Max. $J_{\phi}$ \\
\hline Spectral & $8.75 \times 10^{-4}$ & -7.33 & $3.324 \times 10^{-2}$ & $-1.624 \times 10^{-2}$ & 0.1371 \\
\hline 7.0 & $8.37 \times 10^{-4}$ & -7.47 & $3.202 \times 10^{-2}$ & $-1.449 \times 10^{-2}$ & 0.1449 \\
12.3 & $8.48 \times 10^{-4}$ & -7.42 & $3.237 \times 10^{-2}$ & $-1.492 \times 10^{-2}$ & 0.1370 \\
23.0 & $8.50 \times 10^{-4}$ & -7.41 & $3.244 \times 10^{-2}$ & $-1.536 \times 10^{-2}$ & 0.1370 \\
\hline
\end{tabular}

Table 5. Magnetic energy $E_{\text {mag }}$, its growth rate $k$, and local magnetic field for $t=100$.

\begin{tabular}{c|cccc}
\hline$r_{m}$ & $E_{\text {mag }}$ & $k$ & $B_{z}$ at $\boldsymbol{r}=\left(0,0, r_{o}\right)$ & $B_{z}$ at $\boldsymbol{r}=\left(r_{o}, 0,0\right)$ \\
\hline Spectral & $4.69 \times 10^{-3}$ & $-1.56 \times 10^{-2}$ & $4.20 \times 10^{-2}$ & $-2.34 \times 10^{-2}$ \\
FEM & $4.77 \times 10^{-3}$ & $-1.58 \times 10^{-2}$ & $4.12 \times 10^{-2}$ & $-2.25 \times 10^{-2}$ \\
\hline
\end{tabular}
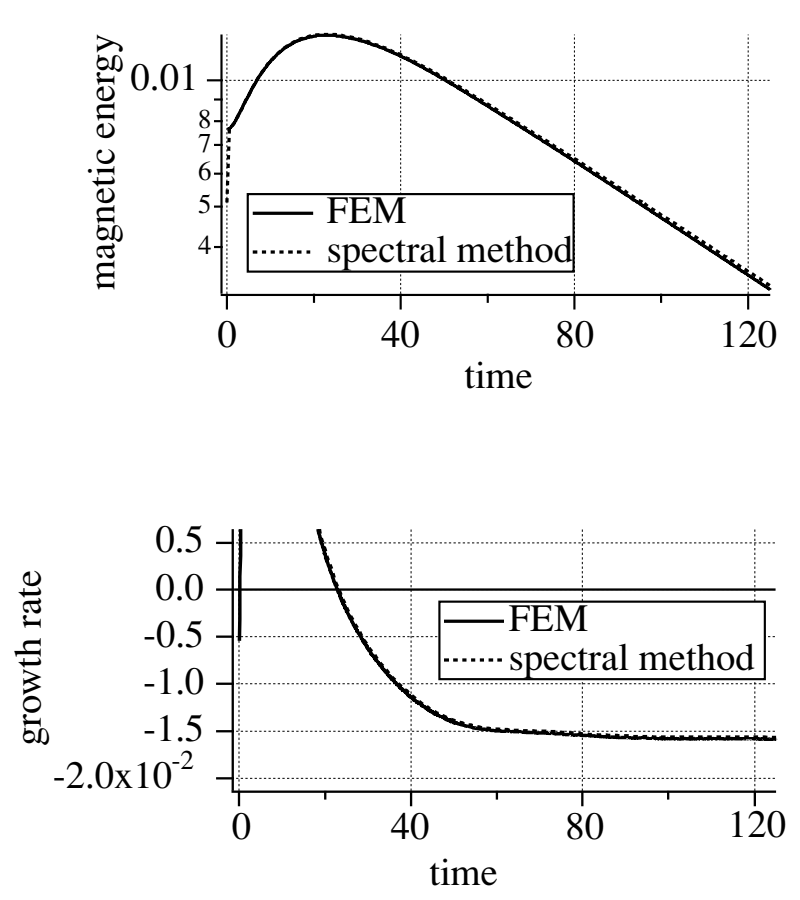

Fig. 8. Time evolutions of the averaged magnetic energy (upper panel) and the magnetic energy growth rate (lower panel) for the kinematic dynamo problem.

1970), which treated a sphere without the inner core. Here the inner core is incorporated. In the present model, the velocity field in the shell is given by

$$
\begin{aligned}
\boldsymbol{u}= & \nabla \times \nabla \times\left\{\left(U_{S 2}^{0}+U_{S 2}^{2 s}+U_{S 2}^{2 c}\right) \hat{r}\right\} \\
& +\nabla \times\left(U_{T 1}^{0} \hat{r}\right) \\
U_{T 1}^{0}= & 0.5\left(r-r_{i}\right)^{2}\left\{1-\left(r-r_{i}\right)^{2}\right\} Y_{1}^{0},
\end{aligned}
$$

$$
\begin{aligned}
& U_{S 2}^{0}=0.08\left(r-r_{i}\right)^{6}\left\{1-\left(r-r_{i}\right)^{2}\right\}^{4} Y_{2}^{0}, \\
& U_{S 2}^{2 s}=0.21\left(r-r_{i}\right)^{4}\left\{1-\left(r-r_{i}\right)^{2}\right\} Y_{2}^{2 s},
\end{aligned}
$$

and

$$
U_{S 2}^{2 c}=0.21\left(r-r_{i}\right)^{4}\left\{1-\left(r-r_{i}\right)^{2}\right\} Y_{2}^{2 c}
$$

The initial value for the vector potential is the same as that used in the magnetic diffusion test. We use Mesh (h) as described in Table 3 with $r_{m}=12.0$. The magnetic diffusivity $\eta$ is $1 / 500$ in the present simulation. As in the previous test of the magnetic diffusion, the results are compared with the results of the spectral technique. In Fig. 8, we plot the temporal evolution of the magnetic energy and its growth rate. The magnetic energy increases at the beginning of the simulation and decays at the end of the simulation. This behavior is observed for both solutions. However, the magnetic energy in the FEM case is slightly smaller, and diffuses slightly faster than that in the spectral method case throughout the simulation. Since similar behavior is also observed in the magnetic diffusion test, this difference may be caused by the treatment of magnetic diffusion. However, these errors are small: The difference in the growth rates of the two cases is only $1.3 \%$ as seen in Table 5.

Snapshots of the magnetic field and current density for $t=100$ are given in Fig. 9. The patterns of the magnetic field and current density are almost the same in the two cases. However, some current density is seen outside the fluid shell in the FEM case. This small current is a numerical artifact resulting from the spatial discretization. In Table 5, the $z$ component of the magnetic field at specific points is given. The magnetic field differs by approximately $4 \%$ between the two cases at the equatorial plane. 


\section{FEM}

\section{magnetic field}

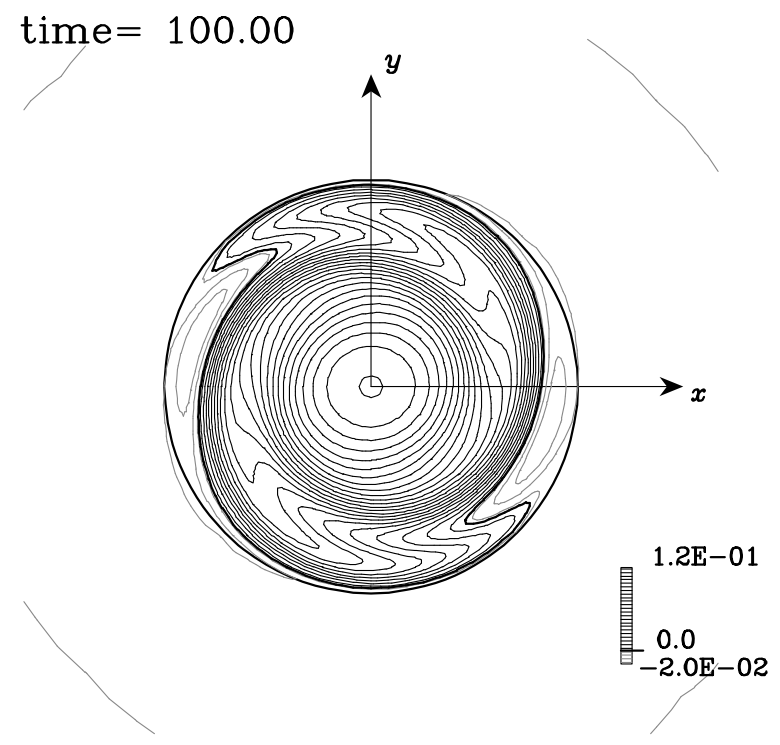

\section{current density}

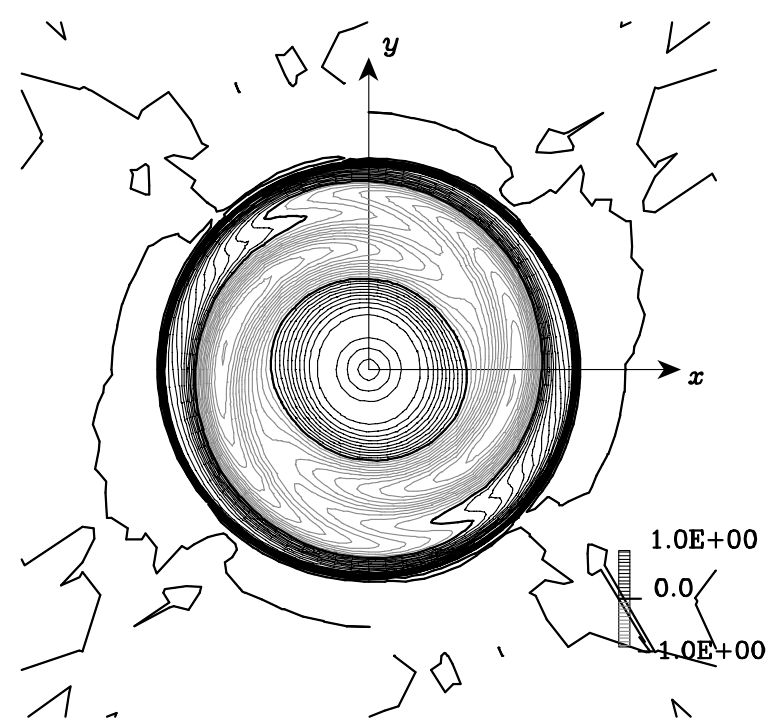

\section{Spectral method}

magnetic field

time $=100.00$

$\mathrm{z}=0.750$

\section{current density}
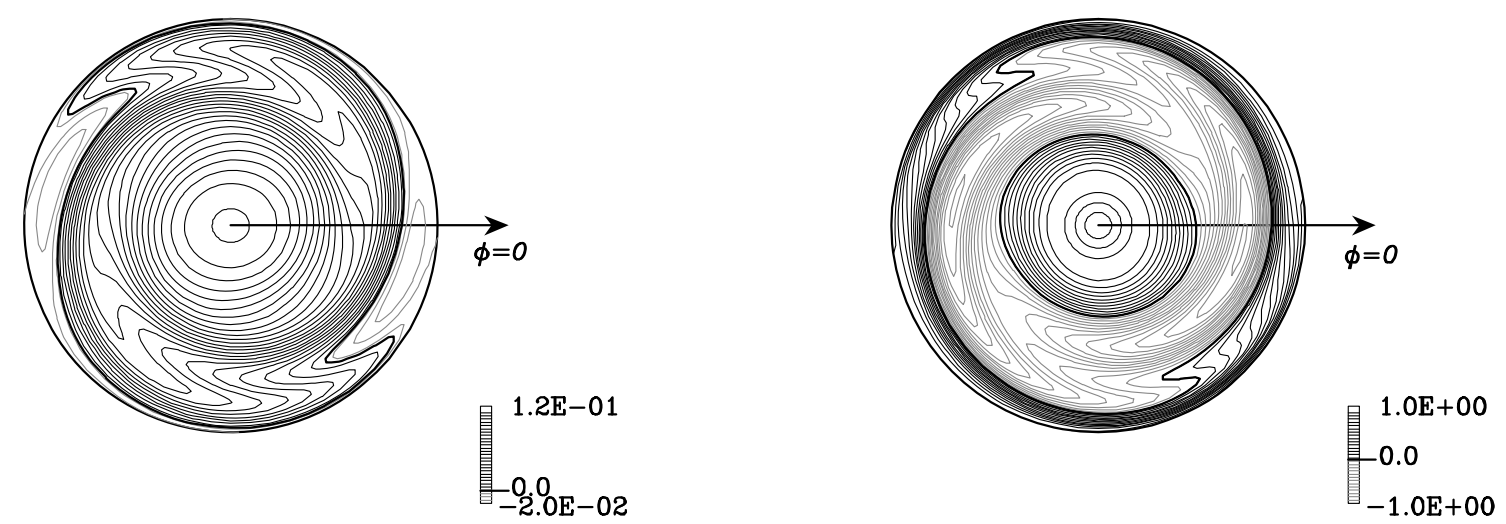

Fig. 9. Intensity of the $z$-component of the magnetic field (left panels) and the current density (right panels) in a cross section at $z=0.75$ for $t=100.0$. The FEM result is given in the upper panels, and the results of the spectral method are given in the lower panels.

\section{Discussion}

As seen in the previous sections, the simulation approach described here can represent the magnetic field to within 5\% of the analytical solutions or results obtained by the spectral method. As described in Section 1, Chan et al. (2001a, b) have developed a similar simulation code for the geodynamo process. There are some principal differences as listed in Section 1. Chan et al. also verified their model using the magnetic diffusion and the kinematic dynamo model of Zhang and Busse (1989). The model of Chan et al.'s model has higher accuracy than the present model. For example, $r_{m}>9.67=5.8 r_{o}$ is required for the simulation domain in the present study to obtain sufficient accuracy while only $r_{m}=3 r_{o}$ is required in the other model. However, in Chan et al.'s model, insulator is replaced with a region of large magnetic diffusivity and the field is determined by solving the magnetic diffusion equation. The most significant advantage in the present model is that it can accommodate an electrical insulator outside the fluid shell. We find small errors in the present results from the spectral solution. How- 
ever, we consider that the accuracy of the FEM solution will be increased by using a finer finite-element mesh than the one utilized here. In addition, the introduction of infinite elements may improve accuracy since $B_{z}$ at the equator was found to exhibit larger errors than those found with other solution techniques, and this error decreases with increasing $r_{m}$ (see Table 4).

We consider a treatment of boundaries between a conductive fluid and an insulator in the present study. The present numerical program can treat any magnetic diffusivity. The mantle can be either insulating or conducting. The inner core can also have different diffusivity from that of the outer core. In that case, Eq. (34) is solved with different magnetic diffusion $\eta$ for each layer. We plan to perform numerical simulations with different inner core diffusivity from that in the outer core as a future study.

\section{Conclusion}

We have developed a code for MHD simulation in a rotating spherical shell using a parallel FEM platform. With this technique, tri-linear hexahedral elements are chosen for the spatial discretization of both the conducting fluid shell and the exterior insulator. All physical values are interpolated using tri-linear functions. The vector potential of the magnetic field with the Coulomb gauge is computed to obtain the magnetic field, and the potential is determined to satisfy the vector potential conservation law. For time integration, we use the fractional step scheme; the Crank-Nicolson scheme is used for the diffusion term, and the 2nd-order Adams-Bashforth scheme is used to solve the other terms. The Laplace equation for the vector potential is solved in this process simultaneously. The vector potential is set to zero at the outer boundary of the simulation domain $r_{m}$, instead of the real boundary condition at an infinite radius. Our results suggest that $r_{m} \geq 9.667=5.8 r_{o}$ is required to obtain adequate accuracy for the dipole field. The result also suggest that the radial resolution around the boundary between the fluid and the insulator is more important than the size of the simulation domain in determining the accuracy of the higher modes of the magnetic field mode. Throughout the present tests, the differences between the results of the FEM calculation and a spectral method are approximately $2 \%$, except for the $z$-component of the magnetic field at the equator. This component has errors as large as $5 \%$ at the equator, but we consider that in order respects the new simulation code can accurately represent the magnetic field.
Acknowledgments. This study is part of the "Solid Earth Platform for Large-Scale Computation" project funded by the Ministry of Education, Culture, Sports, Science and Technology, Japan, through the Special Promoting Funds of Science \& Technology. The authors would like to thank Dr. Yoshitaka Wada for his work on mesh generation, and our colleagues in the GeoFEM project team for their support.

\section{References}

Bullard, E. C. and H. Gellman, Homogeneous dynamos and terrestrial magnetism, Proceedings of Royal Society of London, A247, 213-278, 1954.

Chan, K. H., K. Zhang, J. Zou, and G. Schubert, A nonlinear vacillating dynamo induced by an electrically heterogeneous mantle, Geophysical Research Letters, 28, 4411-4414, 2001a.

Chan, K. H., K. Zhang, J. Zou, and G. Schubert, A nonlinear 3-D spherical $\alpha^{2}$ dynamo using a finite element method, Geophysical Research Letters, 28, 4411-4414, 2001b.

Christensen, U., P. Olson and G. A. Glatzmaier, Numerical modelling of the geodynamo: A systematic parameter study, Geophys. J. Int., 138, 393409, 1999.

Clune, T. C., J. R. Elliott, M. S. Miesh, J. Toomre, and G. A. Glatzmaier, Computational aspects of a code to study rotating turbulent convection in spherical shells, Parallel Computing, 25, 361-380, 1999.

Glatzmaier, G. A. and P. H. Roberts, A three-dimensional self-consistent computer simulation of a geomagnetic field reversal, Nature, 377, 203209, 1995a.

Glatzmaier, G. A. and P. H. Roberts, A three-dimensional convective dynamo solution with rotating and finitely conducting inner core and mantle, Physics of Earth and Planetary Interiors, 91, 63-75, 1995b.

Kageyama, A., T. Sato, and the Complexity Simulation Group, Computer simulation of a magnetohydrodynamic dynamo, Physics of Plasmas, 2, 1421-1431, 1995.

Kuang, W. and J. Bloxham, An Earth-like numerical dynamo model, Nature, 389, 371-374, 1997.

Lilley, F. E. M., On kinematic dynamos, Proceedings of Royal Society of London, A316, 153-167, 1970.

Matsui, H. and H. Okuda, Thermal convection analysis in a rotating shell by a parallel finite-element method-development of a thermal-hydraulic subsystem of GeoFEM, Concurrency and Computation: Practice and Experience, 14, 465-481, 2002.

Nakajima, K. and H. Okuda, Parallel iterative solvers with localized ILU preconditioning for unstructured grids on workstation cluster, International Journal of Computational Fluid Dynamics, 12, 315-322, 1999.

Sakuraba, A. and M. Kono, Effects of the inner core on the numerical simulation of the magnetohydrodynamic dynamo, Physics of Earth and Planetary Interiors, 111, 105-121, 1999.

Zhang, K. K. and F. H. Busse, Convection driven magnetohydrodynamic dynamos in rotating spherical shells, Geophysical and Astrophysical Fluid Dynamics, 49, 97-116, 1989.

H. Matsui (e-mail: matsui@geosci.uchicago.edu) and H. Okuda 